\title{
Reasons for noncompliance with five-yearly screening flexible sigmoidoscopy
}

\section{Charlie Henri Viiala John Kevin Olynyk}

School of Medicine and Pharmacology, Fremantle Hospital, University of Western Australia, Perth, Western Australia, Australia
Correspondence: Charlie Viiala Department of Gastroenterology, Sir Charles Gairdner Hospital, Hospital Avenue, Nedlands, WA, Australia 6009

$\mathrm{Tel}+61893463677$

Fax +6I 893463207

Email charliev@iinet.net.au
Objective: To determine factors influencing the low return rate observed in a program of flexible sigmoidoscopy for average risk screening for colorectal carcinoma.

Methods: Flexible sigmoidoscopy-based screening of average risk 55-64 yr olds has been ongoing since 1995. Greater than 3400 primary and 1000 follow up screening examinations have been performed. Participants with a primary screen in 1997-1999 and eligible for rescreening in 2002-2004 were studied. A questionnaire assessing possible reasons for noncompliance was sent to subjects who did not attend the five year repeat screening.

Results: 1672 primary screening flexible sigmoidoscopies were performed in 1997-1999 with 1362 being normal or having hyperplastic polyps only. The return rate was 45\%: 48\% of eligible males and $39 \%$ of eligible females had returned ( $p=0.001$ for difference). 709 questionnaires were mailed with a $50 \%$ response rate and 162 requests for repeat flexible sigmoidoscopy were generated. $27 \%$ of all respondents had undergone further bowel evaluation since the original normal sigmoidoscopy. Of eligible subjects who refused further screening, $65 \%$ did so because of concerns over procedural pain.

Conclusions: Reasons for nonattendance relate to uptake of other bowel investigations and pain felt at initial screening. Return rate can be raised with ongoing prompting to attend screening. Keywords: sigmoidoscopy, colonoscopy, colonic neoplasm, population surveillance, patient compliance

\section{Introduction}

Five-yearly flexible sigmoidoscopy (FS) is endorsed by many health authorities as an option for screening asymptomatic average risk individuals for colorectal cancer (Winawer et al 2001; Australian Cancer Network Colorectal Cancer Guidelines Revision Committee 2005). The effectiveness of this screening pathway will be influenced by participation rates for initial screening and subsequent follow up. This is particularly relevant for a screening modality that may only have a moderate sensitivity. Using the example of fecal occult blood testing (FOBT), a meta- analysis of randomized trials of FOBT reported an overall $16 \%$ mortality reduction from biennial screening but with a $23 \%$ reduction in those actually complying with screening (Towler et al 1998). In trials of flexible sigmoidoscopy, widely varying initial participation rates have been reported, ranging from 15\%-81\% (Hoff et al 1985; Olynyk et al 1996; Verne et al 1998). Factors such as perceived lack of risk (either by lack of symptoms or family history), practical obstacles to undertaking the test, and fear or embarrassment regarding FS have been identified as significant barriers to attendance (Vernon 1997; McCaffery et al 2001). Concern about procedural pain or embarrassment may be more significant for females compared to males (Weinberg et al 2004). Lack of interest or time is a commonly cited reason for nonattendance (Olynyk et al 1996). Additionally, in a randomly selected cohort, up to $30 \%$ of nonattendees were found to be ineligible for average risk screening (Olynyk et al 1996). 
There is relatively little data examining the reasons for drop out of a program of serial flexible sigmoidoscopy. A community based flexible sigmoidoscopy screening program has been in operation at Fremantle Hospital, Western Australia since 1995. Since inception, over 3400 screenings of average risk individuals have been performed and over 1000 have returned for 5 year follow up, with an apparent return participation rate of $45 \%$ of eligible subjects. In this paper, we investigate the reasons why over half the subjects invited for repeat screening do not attend.

\section{Methods}

In 1995, a program of unsedated flexible sigmoidoscopybased screening of asymptomatic average risk individuals aged 55-64 years was established at Fremantle Hospital. The project was conceived as a pilot study of this screening modality in Australia and was offered as a free service. At the time, no formal CRC screening program existed in the country and only recently has a national FOBT based program commenced. In addition to FOBT, Australian national guidelines include five-yearly FS as a screening option and the procedure can be accessed through public or private medical providers (Australian Cancer Network Colorectal Cancer Guidelines Revision Committee 2005).

Letters of invitation were sent to potentially eligible subjects selected randomly from the Western Australian Electoral Roll. The cohort was drawn from postal codes in the geographic vicinity of the hospital. Participation from volunteers was also accepted. The methods and progress results of the screening program have been previously reported (Olynyk et al 1996; Collett et al 2000). In the program overall, $42 \%$ of participants have been volunteers for screening. Insertion depth of the exam and the self reported pain score has been prospectively recorded. The pain score was recorded following the FS by use of a $0-10$ point visual analogue scale (with a higher score reflecting greater pain).

From the year 2000 onwards, five-year recall of subjects with an initially normal (no polyps or hyperplastic polyps only) examination has been performed. Subjects were sent invitations for repeat flexible sigmoidoscopy with a request to contact the program office to accept further screening. This letter is signed by the senior clinician in the program (JO) and outlines the benefits of repeat screening even if the original exam was normal or the subject is asymptomatic. The letter also asked about abdominal symptoms and family history of bowel cancer. People with symptoms or family history were advised to see their local general practitioner. At the time of phone contact, an interview is conducted to ascertain continued eligibility for screening. However, routine questioning regarding previous bowel investigations was not undertaken. Invitations for repeat FS were mailed to the last known address of participants as recorded in a dedicated database. If letters had been previously returned to sender, an effort was made to identify a new address by consulting a centralized hospital encounters database or using any available forwarding address. Persons agreeing to further screening were allocated a booking for FS but this appointment could be up to six months later. Repeat procedures within the program have been performed by the same staff and methods as for the primary examination and were only offered at this institution

The set of persons who underwent primary screening in the years 1997-1999 and were apparently eligible for rescreening in 2002-2004 but did not return were selected as the study group. Screening participants from 1995-1996 were not chosen due to the length of time that has elapsed since their original examinations. Previous participants who were known to be deceased, had known interval colorectal cancer cases, those that had previously requested withdrawal from screening and subjects that had undergone colonoscopy at our institution (determined by reference to a hospital electronic endoscopy database) were excluded from the study. Additionally, central hospital databases were referenced to identify deceased individuals and to update all mailing addresses where possible.

The study group was assessed in two ways. Initially, univariate analysis of factors predicting attendance was performed by chi-square testing for categorical variables and a logistic regression model was used to examine the effect of multiple categorical variables on future attendance of screening where a bivariate association with $\mathrm{p}<0.2$ was observed. Comparison of results between groups was also done with t-tests. Data was analyzed using the Statistical Package for Social Sciences, version 11.0 (SPSS Inc, Chicago, Illinois). The variables considered were: gender, pain score recorded at original examination (score $\leq 3.0$ or $>3.0$ ), proceduralist seniority (consultant or registrar/fellow), proceduralist training background (physician or surgeon) and insertion depth of the original examination.

Secondly, a short anonymous questionnaire was sent to those subjects identified as nonattenders. Information was sought on reasons for nonattendance, listing a series of options which were broadly grouped as; lack of interest, lack of time, other bowel testing, health changes, and dissatisfaction with original examination. Where appropriate, the subject could enter additional information or select from a series of suboptions. A further section asked for the 
individual's opinion on how the screening procedure could be improved. Additionally, an option was given for the participant to request a new booking for follow up FS. All responses received were included in the analysis.

\section{Results}

Between 1997 and 1999, 1672 primary screening flexible sigmoidoscopies were performed. A total of 1362 subjects were considered eligible for a recall examination and had been mailed an invitation to re-attend after 5 years. However, our review of medical records resulted in seventy seven individuals being excluded from the cohort: 12 known cancers (10 of these interval colorectal cancer), 32 deceased subjects, 11 subjects having later colonoscopy at Fremantle Hospital and 22 cases were subjects having already notified their intention to withdraw from the program $(80 \%$ of these were because of new health problems that subjects felt superseded concerns regarding colorectal cancer). At the time of our study, 513 subjects had undergone a second FS and 63 subjects were on a waiting list, yielding an apparent return rate for 5 year follow-up of 576/1285 (45\%) for eligible persons. A flowchart of patient recruitment into the survey is shown in Figure 1.

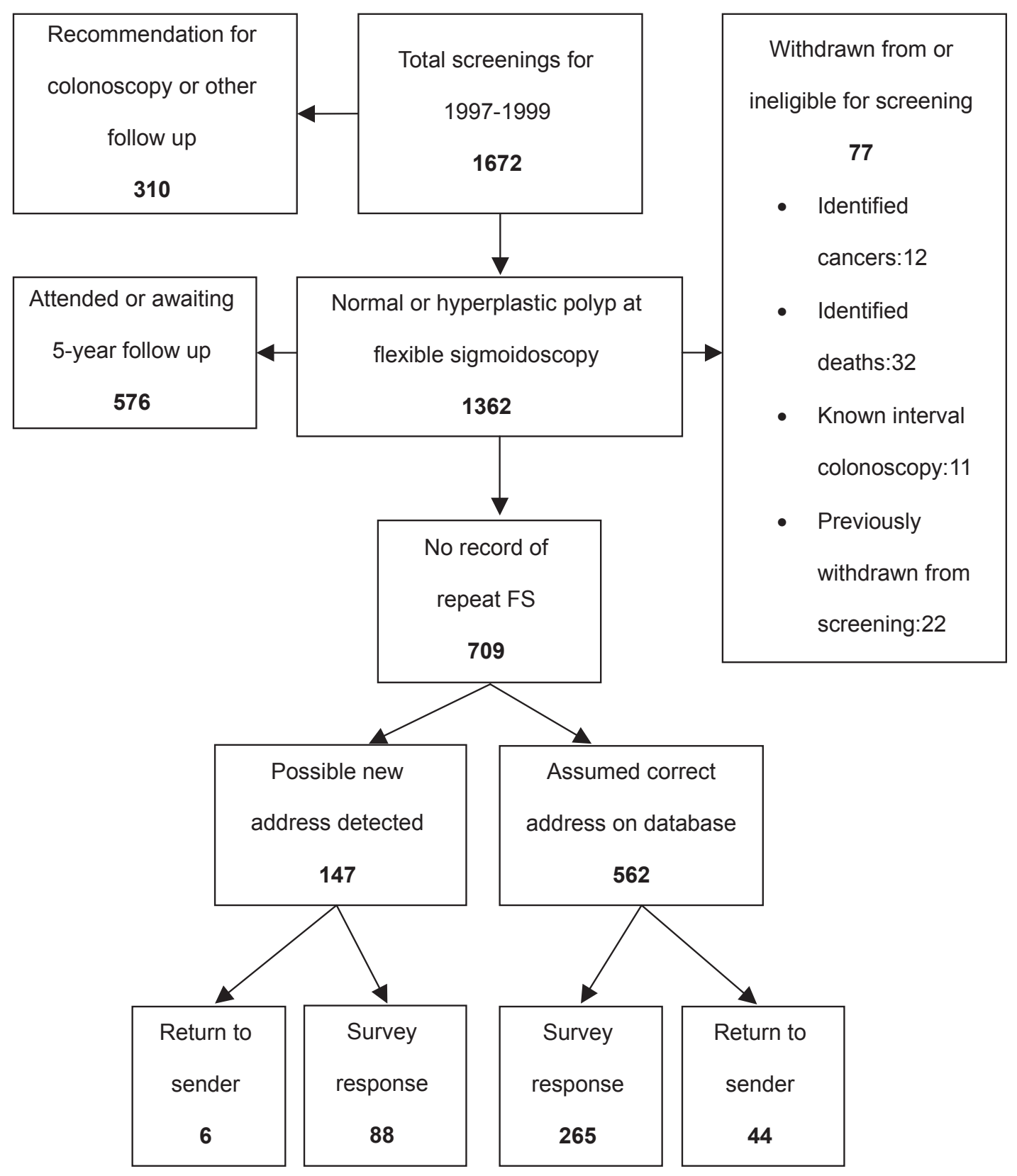

Figure I Flowchart of patient recruitment into the survey. 
Review of the screening database prior to the current study indicated that $48 \%$ of apparently eligible males and $39 \%$ of eligible females returned for follow-up screening ( $p=0.001$ for difference between genders). The mean pain score reported for the primary exam was 3.1 for all those attending follow-up screening compared with 4.0 for those not attending follow-up $(\mathrm{p}<0.0001)$. On univariate analysis, female gender, pain score more than 3.0 , procedure performed by a fellow and insertion depth of less than $50 \mathrm{~cm}$ were associated with a reduced likelihood of return $(p<0.2)$. The training background of the initial proceduralist did not appear significant. Female sex (OR 0.8, 95\% CI 0.6-0.9), pain score more than 3.0 (OR 0.7, 95\% CI 0.6-0.9) and insertion depth less than $50 \mathrm{~cm}$ (OR 0.7, 95\% CI 0.5-0.9) remained as independent predictors of future nonattendance following logistic regression analysis.

After exclusions, 709 nonattending subjects (51\% male) remained eligible for our study: 562 of whom had the same current mailing address whilst 147 had a new address found.
Three hundred and fifty three responses were received $(50 \%$ response rate) with an additional 50 letters returned to sender. Fifty two percent of respondents were female. One hundred and sixty two respondents requested a new booking for FS and were considered eligible to continue in the screening program.

Of 353 respondents, 94 (27\%) reported having further bowel evaluation in the time since their original FS (Figure 2). Forty three percent indicated that this was because of new bowel symptoms and $8 \%$ because of a new family history of CRC, although almost half of respondents did not give a specific reason. Colonoscopy was the investigation performed in at least 48 cases though once again, many did not specify the investigation performed in their response.

Ninety seven (28\%) of the 353 respondents definitely declined further participation in FS screening and had not undergone any other interval bowel evaluation. When asked to provide reasons for this decision, $65 \%$ cited the pain or unpleasantness of FS as a key determinant (Figure 2) with a

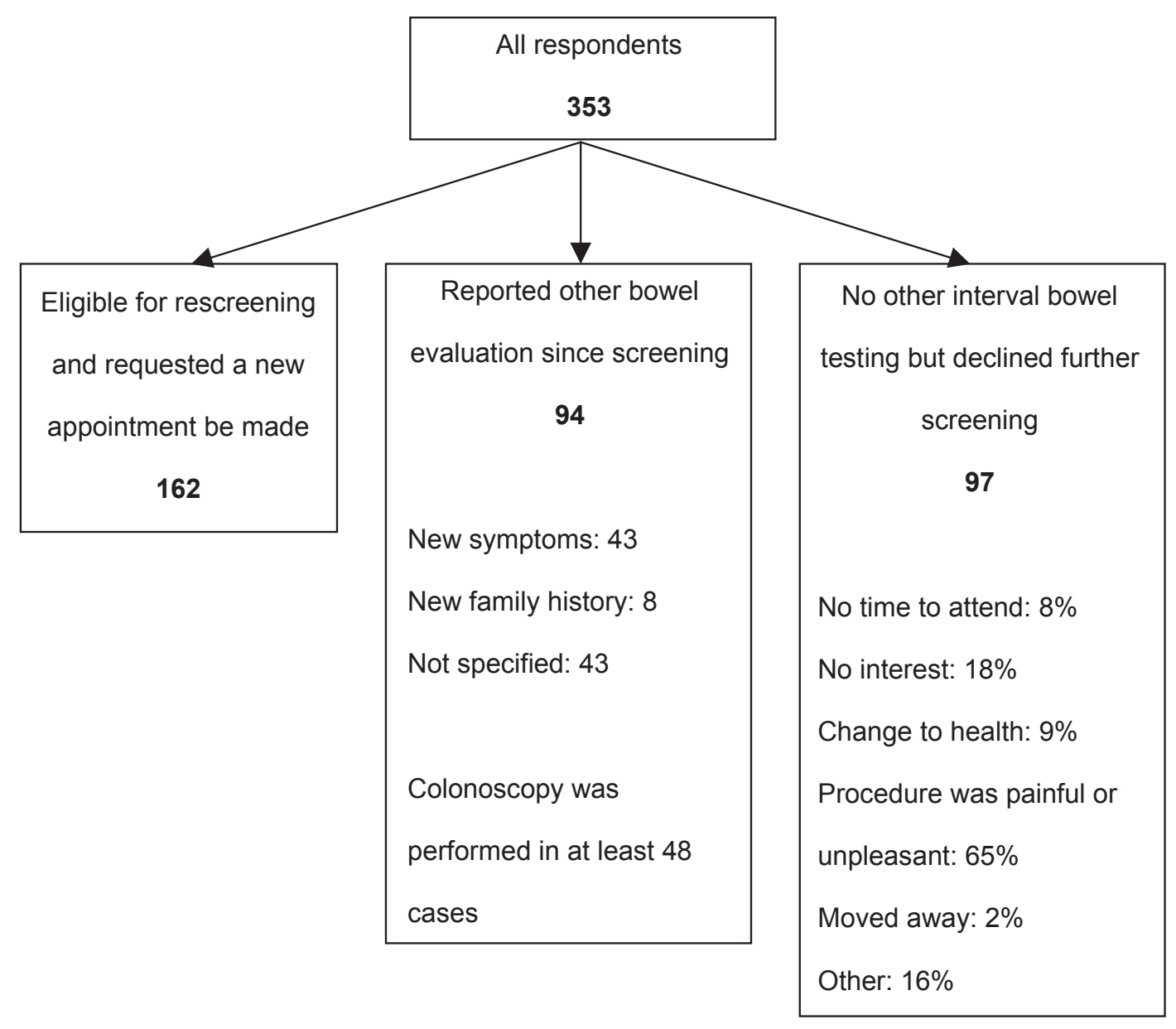

Figure 2 All respondents' reasons for no further attendance of serial flexible sigmoidoscopy. 
similar proportion of males and females giving this response. Lack of interest or lack of time was a less frequent reason. Nine percent listed a major health change with examples of malignancy or cerebrovascular disease being cited. Other reasons provided included feelings of embarrassment, perceptions of inexperienced or uncaring staff and poor communication of results following the procedure.

Respondents were asked to comment on ways to improve the experience they had of FS screening and 153 gave a response. Not surprisingly, 139 (91\%) indicated that measures to reduce pain were required. More flexibility of appointment times $(9 \%)$, less time in hospital on the day $(8 \%)$ and less delay to appointment dates $(3 \%)$ were also selected. A number of respondents wrote that they would elect to receive anesthesia at their next FS, indicating some failure on the program's part to communicate the nature of the screening procedure adequately.

\section{Discussion}

Flexible sigmoidoscopy every 5 years is recommended as an option for screening average risk individuals for colorectal cancer although screening trials are currently investigating the impact of once only screening FS (Segnan et al 2002; UK Flexible Sigmoidoscopy Screening Trial Investigators 2002). Our program has previously reported that $10 \%$ and $2 \%$ of repeat examinations find neoplasia and advanced neoplasia, respectively (Viiala et al 2007). Although the uptake of FS in trials is variable, experience suggests that participants find it an acceptable procedure and the majority of subjects indicate willingness to undergo the procedure again (Nicholson and Korman 2005). Within our own program, 94\% of subjects having an initial FS would have the procedure again (Collett et al 2000). However, the actual return rate of $45 \%$ we have experienced in our program implies that other factors develop over time to reduce participation.

An important finding is that over time, a substantial proportion of subjects change address or may be temporarily absent and do not receive and respond to mailed invitations. Our study found that by offering the option of repeat examination with the questionnaire, we were able to generate 162 bookings for follow up from 709 mail outs. This in itself would theoretically lift the overall return rate from $45 \%$ to $57 \%$. While we had relied on being notified of change of address by program participants, it is clear that a formal screening program requires a mechanism of tracking address changes more effectively, such as by linkage to an electoral roll. The fact that we received 50 returned letters from incorrect addresses even after using hospital databases supports this.
Another finding of significance is the relatively high dropout rate due to uptake of other bowel examinations, with 94/353 (27\%) of respondents reporting further bowel investigation since their original FS. In many, this was due to the development of new symptoms or new family history of CRC and colonoscopy appears to be the investigation of choice. Significant self-rated changes to health, such as stroke or malignancies were reported in 9/97 cases of those who appeared eligible for further screening but declined to have it. It may not be accurate to extrapolate these findings to the entire cohort given our $50 \%$ response rate, but at the very least, some $13 \%$ of the original screening group have died or become ineligible to continue as average risk CRC screening in the interval between 5-yearly FS. If we do assume that similar frequencies of health changes occurred in those who did not reply to our questionnaire and if the new screening requests $(n=162)$ generated by the questionnaire are included, and the potential number of medical exclusions are considered, it is conceivable that up to $21 \%$ of the original participants become ineligible for further "average risk" screening at 5 years. As such, the true participation rate of eligible subjects in ongoing FS screening rises from the observed $45 \%$ rate to $68 \%$. This compares favorably with FOBT trials that report compliance rates with later rounds of screening in the order of 50\%-60\% (Hardcastle et al 1996; Kronborg et al 1996; Mandel et al 1999; Faivre et al 2004).

Analysis of pain score data prior to sending the questionnaire had suggested that the discomfort of FS would be a major determinant of further attendance and that females would be less likely to return. In studies of primary screening by sigmoidoscopy or colonoscopy there has been an indication that women are less likely to attend, despite a large body of literature showing that females are more likely to undertake preventative health measures in general (Senore et al 1996; Seeff et al 2004). A number of studies have reported that females invited for flexible sigmoidoscopy had a number of perceived barriers to screening including anticipation of greater discomfort and embarrassment from the procedure than males (Farraye et al 2004; Wardle et al 2005). Additionally, women report a preference for a female proceduralist (Farraye et al 2004; Menees et al 2005).

It is not surprising that for those subjects declining further screening, FS-related pain was a major determinant in $65 \%$ of cases overall, with similar responses from females and males. For both sexes, the main modifiable risk factor for nonattendance clearly seems to be concern over a painful procedure. Ensuring the initial FS is not distressing will promote compliance with later follow up. Other factors that 
might affect attendance at screening, such as wait-time for an appointment or flexibility with screening times were not rated as very important by most respondents. Studies have addressed pain reduction techniques such as distraction (Lembo et al 1998), different calibre endoscopes (Farraye et al 2004) and medications such as inhaled nitrous oxide (Fich et al 1997), sublingual hyoscine (Dumot et al 1998) or oral midazolam (Kuganeswaran et al 1999). There is an issue with over-complicating the procedure, particularly by introducing sedation thereby reducing any advantages of a FS compared to colonoscopy for large scale screening. An obvious strategy would be to direct such individuals to an alternate screening strategy if initial sigmoidoscopy proves limited or excessively painful. Inadequate screening of subjects is associated with an increased risk of colorectal cancer compared with those who undergo adequate examination (Doria-Rose et al 2005).

Our study has sought to examine reasons why people are not compliant with serial flexible sigmoidoscopy. The conclusions we have drawn are based on the answers from the $50 \%$ of subjects who responded to the questionnaire and may not be representative of the whole group. We chose to use anonymous questionnaires in an attempt to maximize the response to the research although this somewhat limited our analysis of results. We were not able to collect detailed information about the nature of new health problems or the reasons why individuals underwent other bowel investigations and so it is not certain that all these individuals had become ineligible for average-risk screening. Additionally, we are not able to distinguish between those who were originally volunteers for screening compared to invited for screening and determine how the act of volunteering affects future compliance.

In conclusion, our study suggests that the participation rate for serial FS can be substantially improved by accurate tracking of address changes and by repeated mail outs to prompt attendance. In fact, failure to track program participants after their initial examination appears to be the single most important factor affecting attendance at five year follow up. The compliance rate with 5-yearly flexible sigmoidoscopy screening has not been previously well documented and our study shows that at least $13 \%$ of eligible subjects may drop out over time due to health changes or uptake of other bowel investigations. Concern over procedural pain was also a major issue contributing to nonattendance for follow-up screening FS for both genders. Measures that promote attendance at first sigmoidoscopy and minimize the discomfort of the test are likely to increase compliance with future screening.

\section{Acknowledgments}

The authors would like to acknowledge the assistance of Ms Andrea Murray in data collection and preparation during this project. The authors report no conflicts of interest.

\section{References}

Australian Cancer Network Colorectal Cancer Guidelines Revision Committee. 2005. Clinical practice guidelines for the prevention, early detection and management of colorectal cancer. Sydney: The Cancer Council Australia and the Australian Cancer Network.

Collett JA, Olynyk JK, Platell CF. 2000. Flexible sigmoidoscopy screening for colorectal cancer in average-risk people: update of a communitybased project. Med J Aust, 173:463-66.

Doria-Rose VP, Newcomb PA, Levin TR. 2005. Incomplete screening flexible sigmoidoscopy associated with female sex, age, and increased risk of colorectal cancer. Gut, 54:1273-8.

Dumot JA, Verzola E, Nicol S, et al. 1998. Sublingual hyoscyamine for patient comfort during screening sigmoidoscopy: a randomized, double-blind, placebo-controlled clinical trial. Gastrointest Endosc, 48:283-6.

Faivre J, Dancourt V, Lejeune C, et al. 2004. Reduction in colorectal cancer mortality by fecal occult blood screening in a French controlled study. Gastroenterology, 126:1674-80.

Farraye FA, Wong M, Hurwitz S, et al. 2004. Barriers to endoscopic colorectal cancer screening: are women different from men? Am J Gastroenterol, 99:341-49.

Farraye FA, Horton K, Hersey H, et al. 2004. Screening flexible sigmoidoscopy using an upper endoscope is better tolerated by women. $\mathrm{Am} \mathrm{J}$ Gastroenterol, 99:1074-80.

Fich A, Efrat R, Sperber AD, et al. 1997. Nitrous oxide inhalation as sedation for flexible sigmoidoscopy. Gastrointest Endosc, 45:10-12.

Hardcastle JD, Chamberlain JO, Robinson MH, et al. 1996. Randomised controlled trial of faecal-occult-blood screening for colorectal cancer. Lancet, 348:1472-7.

Hoff G, Vatn M, Gjone E, et al. 1985. Epidemiology of polyps in the rectum and sigmoid colon. Design of a population screening study. Scand $J$ Gastroenterol, 20:351-5.

Kronborg O, Fenger C, Olsen J, et al. 1996. Randomised study of screening for colorectal cancer with faecal-occult-blood test. Lancet, 348:1467-71.

Kuganeswaran E, Clarkston WK, Cuddy PG, et al. 1999. A double-blind placebo controlled trial of oral midazolam as premedication before flexible sigmoidoscopy. Am J Gastroenterol, 94:3215-19.

Lembo T, Fitzgerald L, Matin K, et al. 1998. Audio and visual stimulation reduces patient discomfort during screening flexible sigmoidoscopy. Am J Gastroenterol, 93:1113-16.

McCaffery K, Borril J, Williamson S, et al. 2001. Declining the offer of flexible sigmoidoscopy screening for bowel cancer: a qualitative investigation of the decision-making process. Soc Sci Med, 53:679-91.

Mandel JS, Church TR, Ederer F, et al. 1999. Colorectal cancer mortality: effectiveness of biennial screening for fecal occult blood. J Natl Cancer Inst, 91:434-7.

Menees SB, Inadomi JM, Korsnes S, et al. 2005. Women patients' preference for women physicians is a barrier to colon cancer screening. Gastrointest Endosc, 62:219-23.

Nicholson FB, Korman MG. 2005. Acceptance of flexible sigmoidoscopy and colonoscopy for screening and surveillance in colorectal cancer prevention. J Med Screen, 12:89-95.

Olynyk JK, Aquilia S, Fletcher DR, et al. 1996. Flexible sigmoidoscopy screening for colorectal cancer in average-risk subjects: a communitybased pilot project. Med J Aust, 165:74-6.

Seeff LC, Nadel MR, Klabunde CN, et al. 2004. Patterns and predictors of colorectal cancer test use in the adult U.S. population. Cancer, 100:2093-103. 
Segnan N, Senore C, Andreoni B, et al. 2002. Baseline findings of the Italian multicenter randomized controlled trial of "once-only sigmoidoscopy"SCORE. J Natl Cancer Inst, 94:1763-72.

Senore C, Segnan N, Rossini FP, et al. 1996. Screening for colorectal cancer by once only sigmoidoscopy: a feasibility study in Turin, Italy. $J$ Med Screen, 3:72-8.

Smith RA, von Eschenbach AC, Wender R, et al. 2001. American Cancer Society guidelines for the early detection of cancer: update of early detection guidelines for prostate, colorectal, and endometrial cancers. Also: update 2001 - testing for early lung cancer detection. CA Cancer J Clin, 51:38-75.

Towler B, Irwig L, Glasziou P, et al. 1998. A systematic review of the effects of screening for colorectal cancer using the faecal occult blood test, hemoccult. $B M J, 317: 559-65$.

UK Flexible Sigmoidoscopy Screening Trial Investigators. 2002. Single flexible sigmoidoscopy screening to prevent colorectal cancer: baseline findings of a UK multicentre randomised trial. Lancet, 359:1291-300.
Verne JE, Aubrey R, Love SB, et al. 1998. Population based randomized study of uptake and yield of screening by flexible sigmoidoscopy compared with screening by faecal occult blood testing. BMJ, 317:182-5.

Vernon SW. 1997. Participation in colorectal cancer screening: a review. J Natl Cancer Inst, 89:1406-22.

Viiala CH, Olynyk JK. 2007. Outcomes after 10 years of a community-based flexible sigmoidoscopy screening program for colorectal carcinoma. Med J Aust, 187:274-7.

Wardle J, Miles A, Atkin W. 2005. Gender differences in utilization of colorectal cancer screening. J Med Screen, 12:20-7.

Weinberg DS, Turner BJ, Wang H, et al. 2004. A survey of women regarding factors affecting colorectal cancer screening compliance. Prev Med, 38:669-75.

Winawer S, Fletcher R, Rex D, et al. 2003. Colorectal cancer screening and surveillance: clinical guidelines and rationale - Update based on new evidence. Gastroenterology, 124:544-60. 
